\title{
PERSONENREGISTER INDEX DES PERSONNES
}

Autorenangaben und Namensnennungen, die nur als Quellenbelege aufgefuihrt sind, wurden nicht berücksichtigt. Äbte, Monnche und andere Geistliche sowie Fürsten und übrige Herrscher sind unter ihrem Vornamen eingeordnet. Bei aus dem Chinesischen transkribierten Namen steht grundsätzlich der Familien- vor dem Vornamen.

Les noms de personnes mentionnés dans les références des sources ne sont pas pris en considération. Les abbés, moines et ecclésiastiques, de même que les princes et souverains sont cités à leur (pré)nom. Pour les noms chinois transcrits en alphabet latin, on a pris en compte le patronyme.

Adalbéron, évêque de Laon $117^{+21}$

Adalbert II, comte de Calw $82^{25}$

Adalbert III, archevêque de Salzbourg $80^{16}$

Adalbert, archevêque de Magdebourg, chroniqueur $94^{+8}$

Adam, châtelain d'Amiens $122^{49}$

Adrien IV, pape $80^{16}$

Alberti, Leon Battista 149, 156, 164

Albomoz, Egidio 160s.

Alexandre II, pape $81^{23}$

Alexandre III, pape $80^{16}, 171$

Alexandre, saint, martyr romain 106

Alexis II Comnène, empereur 62,65 , 68s., 71

Alexis III Ange, empereur $62,64,69$

Alexis IV Ange, empereur 69s.

Alexis le Stoudite, patriarche de Constantinople 66

Alexis V Mourtzouphlos, empereur 69s.

Ambroise, saint, évêque de Milan 30, 142

An, empereur $42^{10}, 43,54$

André II, roi de Hongrie 16

André, abbé de Strumi $132^{5}, 137^{25}, 141^{44}$, 144

Andronic $I^{a}$ Comnène, empereur 62 , 68

Andronic II, empereur 71

Ansel, évêque de Beauvais 118,120

Antonius, évêque de Fussala $26^{33}, 34$, 37

Arany, János $211^{15}$

Arcadius, empereur 28

Ariald, clerc de Milan 137, 141, $144^{\text {t69 }}$

Amulf, auteur milanais $137^{25}$

Arsène, patriarche de Constantinople 68
Augustin, saint, évêque d'Hippone 19, $21^{+8}, 22-24,25^{+27}, 26,28-32,34,35^{+85}$, $36^{+90,93}, 37 \mathrm{~s}$.

Axido, chef de circoncellions $27,28^{42}, 33$

Ball, John 16

Basile, évêque de Césarée 24

Basile $I^{\text {ter }}$ le Macédonien, empereur 65

Basile II le Bulgaroctone, empereur 5861, 65-68

Basina, fille du roi Chilpéric $I^{\text {er }} 85$

Batatzès, Jean, lieutenant de Léon Tormikios 64

Baudri, évêque de Liège 80

Baudry, évêque de Noyon 119s.

Baugulf, abbé de Fulda 101

Béatrix, épouse de Matthias de Hongrie 208

Benno II, évêque d'Osnabrück 107

Benoît de Nursie $76^{+3}, 79,89^{59}$

Benzon d'Alba, chroniqueur 145

Bérenger, fils de Gebhard 93

Berlichingen, Gơtz von 229

Bernard, roi d'Italie 102

Bernhar, abbé de Hersfeld $81^{22}$

Bolingbroke, Henri, voir Henri IV, roi d'Angleterre

Bonfini, Antonio 206

Boniface VIII, pape 153

Boniface IX, pape 162

Boniface, saint, missionnaire $99 \mathrm{~s}$.

Bonizon, cardinal-évêque de Sutri $137^{25}$, $139,141,144$

Bote, Hermann 187-189, 199

Boyer, Arnaud $222^{39}$

Boyer, Jean $222^{39}$ 
Boyer, Pierre $221,222^{+39}, 223,227 \mathrm{~s}$.

Boyer, Raymond $222^{39}$

Bracciolini, Poggio 164

Bré, Pierre de 220, 221 $1^{+32}, 222,228$

Brisson, Barnabé, président du Parlement de Paris 267

Brun Candidus $88^{53}$

Brun, Rudolf, maire de Zurich 195

Brunon, historien saxon 108

Bruyères, Jacqueline de 220

Caccia, Stefano 164

Carle, Guillaume 215s.

Carloman, maire du palais 100

Carloman, roi de Bavière 93

Carretto, Ottone del 165

Cassiodore 25

Celer, sénateur donatiste 34,36

Ch'in Shih-huang-ti 40

Charibert, roi des Francs 85

Charlemagne, empereur $87^{49}, 95,97,99$ $103,105^{57}, 281,283$

Charles le Gros, empereur 94

Charles IV, empereur 156,160

Charles V, roi de France 222

Charles VI, roi de France 222

Charles II, roi de Hongrie, voir

Charles III, roi de Naples

Charles III, roi de Naples 207s.

Charles Martel, maire du palais 100

Charles Robert, roi de Hongrie 206

Cheng Hsüan 51

Chilpéric $\mathrm{I}^{\mathrm{er}}$, roi des Francs 85

Chômatianos, Dèmètrios, archevêque de Bulgarie 71

Chôniatès, Nicétas 69s.

Christophe, abbé de San Pietro al Po 138

Chrodechilde, fille du roi Charibert 85

Cilli, Barbara de 210

Clément VI, pape 157

Commode, empereur romain $31^{63}$

Comnène Doucas, Théodore, despote d'Épire, empereur de Thessalonique 71

Comnène, Anne 69

Conink, Peter 191s.

Conrad II, empereur 134

Conrad III, roi 81

Conrad II, archevêque de Salzbourg $80^{16}$

Constance II, empereur romain 24

Constant $\mathrm{I}^{\mathrm{et}}$, empereur romain $19,27,38$

Constantin $\mathrm{I}^{\mathrm{er}}$, empereur romain $31^{\text {t61 }}, 38$

Constantin V, empereur 63,72
Constantin VII Porphyrogénète, empereur 65

Constantin VIII, empereur 65,67

Constantin IX Monomaque, empereur 60s., 63s.

Constantin X Doucas, empereur 67

Corneilhan, Raymond de 220

Craloh, abbé de Saint-Gall 86

Cramaud, Simon de 222

Csák, Matthieu de 206

Cyprien, saint 33

Dampierre, Gui von 190s., 193

Diceto, Raoul de, chroniqueur 171

Dioclétien, empereur romain $19,26,33$

Donatus, évêque de Bagaï 19, 27

Donatus, diacre et colon 26s., $34^{82}$

Doring, Cord 188

Duranti, Jean-Étienne, président du parlement de Toulouse 267

Eberhard I, archevêque de Salzbourg $80^{16}$ Édouard I $\Gamma^{\text {es }}$, roi d'Angleterre $169^{+10}, 175$, 179

Édouard II, roi d'Angleterre 179

Éginhard, biographe de Charlemagne 98 , 101

Egkart, Cort 239

Eigil, abbé de Fulda $87 \mathrm{~s}$.

Ekkehard IV, maître de l'école de SaintGall $84^{+35,37}, 86$

Élisabeth Kotromanić de Bosnie 207s.

Emhilt, abbesse de Milz 101

Enguerrand, comte de Boves $122^{+49}$

Enzelin, moine de Saint-Gall 86

Erlembald, chevalier milanais 137,140 , $141^{+49}, 143^{58}, 144^{69}, 145$

Erluin, prieur de Lobbes 80 s., 85

Eugène IV, pape 163

Everhelm, abbé d'Hautmont $81^{23}$

Évrard, intendant du vidame d'Amiens 125

Fasir, chef de circoncellions $27,28^{42}$, 33

Fastrade, reine, épouse de Charlemagne 97,101

Fei-ti, empereur 51,54

Filelfo, Francesco 164

Folcuin, moine et diacre de Saint-Bertin, abbé de Lobbes $80^{+17}$

Foulques, évêque de Beauvais 117 
Frédéric I $I^{\text {er }}$ Barberousse, empereur $80^{16}$, $131^{+1}, 146$

Frédéric III, empereur 164,210

Frédéric, abbé de Hirsau $81,82^{25}$

Froissart, Jean 213

Fu Chien, roi des Ti 42

Gaismaier, Michael 197

Gara, Miklós de 207

Garin, évêque de Beauvais $116^{16}, 117$

Gaudry, évêque de Laon $115^{15}, 117,122$, 125

Gaunt, John of 177

Gebhard, comte du Lahngau 93

Gennadius, prêtre à Marseille $26 \mathrm{~s}$.

Georg der Bärtige, duc de Saxe 230

Gérard, abbé de Saint-Médard de Soissons $118^{30}$

Gérard, châtelain de Quierzy $117^{+27}$

Gerber, Erasmus 249

Gerhard, abbé de Saint-Gall $77^{6}$

Gisèle, fille du Saxon Hessi 102

Godefroi III le Barbu, duc de HauteLotharingie 94

Godis, Petrus de 149

Gonesse, Gérard de 129

Gothelon I, duc de Lotharingie 94

Gothelon II, duc de Basse-Lotharingie 94

Gratien, empereur romain 23

Grégoire le Grand, pape $75,76^{+3}$

Grégoire de Nazianze, évêque de Constantinople 30

Grégoire, évêque de Tours 85

Grégoire, Henri 65

Gui II, duc de Spolète $94^{+7}$

Guibert, abbé de Nogent-sous-Coucy $111,114,115^{+15}, 117^{27}, 118^{+33}, 120$, $122,124-126,127^{67}, 146^{79}, 277$

Guillaume ${ }^{\text {er }}$, roi d'Écosse 177 s., 180 , 184

Guillaume, un proche du châtelain de

l'évêque de Laon 126

Guy, comte d'Amiens 117

Hadrien I $I^{\text {er }}$, pape $87^{49}$

Hardrad, comte 95, 98s., 101-103, 276, 281,283

Harnack, Adolf von 26

Heden le Jeune, duc de Thuringe 100

Henri II, empereur $81^{22}, 88$

Henri III, empereur 88

Henri IV, empereur 107
Henri VII, empereur 160

Henri II, roi d'Angleterre 171, 177, $178^{+51}, 180,184,275$

Henri III, roi d'Angleterre 172, 175 , $176^{+45}, 179,183$

Henri IV, roi d'Angleterre 177,182

Henri $I^{\text {er }}$, roi de France 118

Henri III, roi de France 262s.

Henri IV, roi de France 257s., 263, 269

Henri I $I^{\text {er }}$, duc de Bavière 97

Henri le Jeune, fils d'Henri II d'Angleterre $171^{+19}, 177,180$

Herbert, comte de Vermandois 120

Hermann, moine de Reichenau $77^{6}$

Hermann de Tournai 126s.

Hessi, comte saxon 102

Hincmar, archevêque de Reims 93, 108

Honorius, empereur 23s., $36^{+92}, 37 \mathrm{~s}$.

Horwath, Jean de 207-210

Howden, Roger de, chroniqueur 180

Hraban Maur, voir Raban Maur

Hsiao-wu, empereur 43, 50

Hsieh Ling-yün 43

Hsieh You 43

Hsü Long $52^{+22}, 53$

Hsü Tao-fu 45 s., $52^{22}, 55$

Huan Hsüan $45,54^{+24}$

Huan Wen 52, 54

Hui-yüan, moine bouddhiste 51

Immo, abbé de Reichenau $77^{6}$

Infessura, Stefano 163s.

Innocent III, pape 69

Innocent VI, pape 220

Isaac $I^{\text {er }}$ Comnène, empereur 61

Isaac II Ange, empereur 64, 69

Isaac, martyr donatiste $27^{+38}, 33^{77}$

Isidore, évêque de Séville 25

Jean I ${ }^{\text {er }}$ Tzimiskès, empereur $58,66 \mathrm{~s}$.

Jean IV Doucas Lascaris, empereur 68

Jean VI Cantacuzène, empereur 70

Jean sans Terre, roi d'Angleterre 174, 177,181

Jean, duc de Berry 222

Jean I ${ }^{\mathrm{er}}$, comte de Soissons 117

Jean, métropolite de Mélitène 60

Jean de Salisbury, évêque de Chartres $170^{+17}, 171$

Jean Gualbert, abbé de Vallombreuse $139,140^{+43}, 141^{+44}, 144$

Jean le Bel, chroniqueur 214 
Jean, dit le Géomètre 60

Jean l'Orphanotrophe 65

Jean Minutus, cardinal-prêtre de Santa

Maria in Trastevere $136^{20}$

Kalomodios, banquier 62

Kamytzès, Manuel 62

Kanabos 70

Karl IV, voir Charles IV

Karl der Große, voir Charlemagne

Kékauménos, Katakalon, officier 61,66

Kern, Fritz $274^{l}$

Kont, Étienne de Hédervári 209-211

Kopay, noble hongrois, époux de Sebe de Zách $206^{3}$

Kroum, khan 63

Küküllö, Jean de, moine franciscain 206

Ladislas V, roi de Hongrie $210,211^{15}$

Ladislas de Hunyad, frère de Matthias I ${ }^{\text {er }}$ 210

Lagazara, Bartolomeo da 165

Lampert de Hersfeld, chroniqueur 86, 98, $107,146^{79}$

Landulf, clerc de Milan $137,140,141^{+44}$

Lanzo, juge royal à Milan 136, 145

Lao-tzu 41

Lécapène, Basile, parakoimonène 58

Léon III, empereur 57

Léon $V$, empereur 63

Léon VI, empereur 71

Leubovera, abbesse de Poitiers 85

Leuvel, Mathieu de 219

Libanios 32

Liprand, clerc de Milan $141^{49}$

Liu Yü 42, 44-47, 54s.

Liudolf, duc de Souabe $78^{9}, 94^{8}, 95^{10}, 97$

Lodersleben, Guillaume de 108

Lothaire $I^{\text {er }}$, empereur 105,107

Louis le Pieux, empereur $78^{7}, 87,95$, $103,105-107,285$

Louis IV de Bavière, empereur 158

Louis le Germanique, roi de Francie orientale $93,107 \mathrm{~s}$.

Louis le Jeune, roi de Francie orientale $93^{+1}$

Louis VI, roi de France 112

Louis VII, roi de France $112,120^{42}$, $128^{69}, 180$

Louis $\mathrm{I}^{\text {er }}$ le Grand, roi de Hongrie 207

Louis II Jagellon, roi de Hongrie 211

Louis d'Orléans 207
Louis de Durazzo 207

Ludwig der Fromme, voir Louis le Pieux

Lu Chen 51, 53

Lu Chih 51

Lu Hsün $14,17,42^{+10}, 44-46,47^{+16}, 49-51$, $52^{+23}, 54,55^{+25}, 56$

Lu Ku 51

Lu Sung $52^{+23}, 53$

Ma Jung 51

Macaire, commissaire impérial 19 , 27

Macrobius, évêque d'Hippone $26,27^{+38}$, $33^{77}, 36$

Magistros, Thomas 71

Mainard, cardinal-évêque de Silva

Candida $136^{20}$

Maitland, Frederic W. 169

Makrembolitissa, Eudocie 67

Maleïnos, Eustathe, magistre 59

Manetti, Gianozzo 164

Maniakès, Georges, officier 60s.

Manuel $\mathrm{I}^{\mathrm{es}}$ Comnène, empereur 68s.

Marcel, Étienne 215, 218

Marcellinus, tribun 22, 34, 36

Marché, Clarembaud du $116^{20}, 124,126$

Marcianus d'Urga $35^{85}$

Marculus, évêque de Vegesala 33

Marie $\mathrm{I}^{\mathrm{rc}}$, reine de Hongrie $207^{+5}, 208-210$

Martin V, pape 161s.

Mastro, Paolo dello 164

Mathias $\mathrm{I}^{\text {er }}$, roi de Hongrie 205s., 208, 210s.

Mauropous, Jean 64

Maximin le Thrace, empereur romain $34^{84}$

Maximinianus de Carthage, martyr donatiste $27^{+38}, 33^{77}$

Maximinianus, évêque de Bagaï $35^{85}$

Médicis, Salvestro de 218

Méginhar, comte, père de Réginhard 102

Méginhard, moine de Fulda 106

Ménandre, comte d'Afrique 31

Méthode, patriarche de Constantinople 72

Mezzabarba, Pierte de 139

Michel I $\mathrm{I}^{\mathrm{er}}$ Rhangabe, empereur 63

Michel III, empereur 65

Michel IV le Paphlagonien, empereur 60, 65

Michel V le Calfat, empereur $60,65 \mathrm{~s}$.

Michel VI Bringas, empereur 61s.

Michel VII Doucas, empereur 67

Michel VIII Paléologue, empereur 68, 73 
Mainier le Monétaire, bourgeois d'Amiens 125

Milon le Tonloyer, bourgeois d'Amiens 125

Monte, Frédéric de 108

Montferrat, Boniface de 70

Montfort, Simon de $175,176^{+45}, 178$ s.

Montpezat, Bérenger de 220

Neumarkt, Jean de 156

Neville, Peter de 172

Newton, John, chevalier 173

Nicéphore ${ }^{\text {er }}$, empereur 63

Nicéphore II Phocas, empereur 60s., $67^{+30}, 68$

Nicolas V, pape 158,164

Nithard, chroniqueur, petit-fils de Charlemagne 105-107

Norbert, abbé de Saint-Gall 84

Notker le Lippu, moine de Saint-Gall 84

Optat, évêque de Milev 21, 26-29, 32

Orkhan, émir ottoman 70

Otton $\mathrm{I}^{\text {er }}$, empereur $78^{9}, 86,95^{10}, 97$

Otton de Northeim, duc de Bavière 107

Otton, évêque de Freising $131,132^{+5}$, $133^{+6}, 134,136,146^{+79}$

Pachôme, saint 24

Pacotte, Dom $221^{34}$

Paraspondylos, Léon, ministre 61

Pareto, Vilfredo 13

Paul, commissaire impérial 19,27

Pépin le Bossu, fils de Charlemagne 97s., $101^{43}$

Pétilien, évêque donatiste 37

Petrarca, Francesco 149, 155

Pétrônas, beau-frère de Michel III 65

Philippe de Souabe, empereur 69

Philippe IV le Bel, roi de France 190-192

Phocas, Bardas 58-61, 68

Phocas, Léon $67^{+30}$

Piccolomini, Enea Silvio, voir Pie II

Pie II, pape 165

Pierre de Blois 275

Platina, Bartolomeo 162, 164

Pollock, Frederick 169

Pons, abbé de Saint-Médard de Soissons $118^{+30}$

Poppon, abbé de Stavelot 84

Porcari, Stefano 16, 149-151, 153-156, $162-166,278$

Possidius, évêque de Calama $22,28,32$
Potho, abbé lombard $87^{+49}$

Primus, colon et sous-diacre $25,27,34^{82}$

Psellos, Michel 59-61, 64s., 67, 71

Purchard, moine de Reichenau $77^{6}$

Raban Maur, abbé de Fulda, archevêque de Mayence 87s., 108s.

Rainfroi, abbé de Saint-Wandrille $81^{19}$

Ranulf, earl de Chester 177s.

Raoul de Fougères $178^{51}$

Ratgaire, abbé de Fulda $81^{20}, 83^{29}, 87 \mathrm{~s}$.

Réginaire, comte 80

Réginhard, complice de Bernard d'Italie, arrière-petit-fils d'Hardrad 102

Réginon, abbé de Prüm $82^{+26}, 94^{8}$

Renaud, abbé de Saint-Médard de Soissons $118^{+30}$

Restitutus, prêtre donatiste $35^{88}$

Richard I ${ }^{\text {et }}$, roi d'Angleterte 184

Richard II, roi d'Angleterre 173, 176s., 183

Rienzo, Cola di 15s., 149-151, 153-162, 165s., 278s.

Robert de Béthune, comte de Flandres 190, 193

Robert, earl de Leicester $177 \mathrm{~s}$.

Robert le Mangeur, bourgeois de Laon 126

Rogatus, évêque d'Assuras $35^{88}$

Rohrbach, Jakob 249

Romain IV Diogène, empereur 67

Romano, Anonimo 149, 156, 160

Routpert, moine de Reichenau $77^{6}$

Salvius, évêque donatiste 30

Sassoferrato, Bartolo de, juriste 154

Sciarra Colonna, baron romain 153

Seranus, proconsul d'Afrique 30

Servus Dei, évêque de Thubursicum Bure $35^{85}$

Shih $\mathrm{Hu} 51,53$

Shun, ministre et successeur de Yao 40

Sigismond de Luxembourg, empereur 16 , 207-211

Simon, meneur d'émeute à Soissons 122

Sklèraina, Marie 60

Sklèraina, Romain, frère de Marie 60

Sklèros, Bardas 58s.

Skylitzès, Jean, historien byzantin $58 \mathrm{~s}$., 63

Smaragde, abbé de Saint-Mihiel $79^{1 !}$

Spondius, procurateur 36

Staurakios 63 
Stefaneschi, Martino 159

Sturmi, abbé de Fulda 100

Sun En $14,17,42^{+10}, 43,44^{+13}, 46,47^{16}$, 48-52, 54-56

Sun Hsiu 50

Sun T'ai, disciple et successeur de Tu 43s., 49

Sunnenberg, Ambrosius 188

Syméon le Stylite, saint 30

Szilágyi, Mibály 210

Szu-ma Lun 50

Szu-ma Tao-tzu 43

Szu-ma Yủan-hsien $43,54^{+24}$

Tassilon III, duc de Bavière 100

Taurinus, comte de l'armée d'Afrique 22, $27,28^{+42}, 29,31,33$ s.

Tertullien 32

Thégan, biographe de Louis le Pieux 102 s.

Théodora Porphyrogénète $61,65,72$

Théodore, higoumène du monastère du Stoudios 63

Théodoret, évêque de Cyr 33

Théophane, chroniqueur 63

Théophanô, épouse de Nicéphore II 67

Théophylacte, fils aîné de Michel I $I^{\text {es }} 63$

Theudegaud, meurtrier de Gaudry 125

Thierry, Augustin $113^{8}$

Thomas, duc de Lancaster 177, 179

Thurócz, Jean de 205, 2064, 207-211, 282

Tiburzio, neveu de Porcari 165

Tissière, Bernard $221^{+34}, 222 \mathrm{~s}$.

Tornikios, Léon 64

Tranchedini, Niccolo 165

Ts'ao P'i 40

Ts'ao Ts'ao 51

Tu, maître taoïste 43

Tyler, Wat 173,183

Umur, émir d'Aydin 70

Uto, fils de Gebhard 93

Valentinien, empereur romain 24

Valla, Lorenzo 156, 162, 164
Van Artevelde, Jacques 218

Van dem Damme, Tile 188s.

Vecchi, Francesco 160

Victor, moine de Saint-Gall 86

Vladimir, prince de Kiev 68

Walsingham, Thomas, moine de SaintAlban 172s., 181

Walter, Robert Fitz 174s.

Walworth, William, maire de Londres 173

Wang Hsün, ministre 43

Wang Kung 43

Wang Mang 40

Wang Ning-chih 49

Wang Tan 54

Weber, Max 274

Werner II, comte entre Enns et Wienerwald 93

Wibald, abbé de Corvey et de Stavelot $25^{27}, 81$

Widerat, abbé de Fulda 86

Widukind, chef saxon 106

Widukind, moine de Corvey 97

Wilk, Hans 239

Willibrord, évêque missionnaire 100

Willo, abbé d'Ebersmünster 88

Winterthur, Johann von 195

Witigowo, abbé de Reichenau $77^{6}$

Wolfhere, chanoine de Hildesheim $81^{22}$

Woodstock, Thomas de, duc de

Gloucester 176

Wu Ning-chih 45

Wu Yin-chih, gouverneur 54

Yahya d'Antioche $67 \mathrm{~s}$.

Yao, souverain mythique chinois 40

Yuan, empereur 51, 53

Yves, comte d'Amiens 117

Yves, évêque de Chartres 119

Zách, Félicien de 206, 210

Zách, Klara de, fille de Félicien $206^{3}$

Zách, Sebe de, fille de Félicien $206^{3}$

Zoé la Porphyrogénète $60,65 \mathrm{~s}$.

Zônaras, Jean 71 